\title{
Detection of fecal interferon-induced transmembrane protein messenger RNA for colorectal cancer screening
}

\author{
CHIE MIYAMOTO $^{1}$, NOBUKI MIYAMOTO ${ }^{1}$, HIROYUKI YAMAMOTO ${ }^{1}$, \\ $\mathrm{KOHZOH}$ IMAI $^{2}$ and YASUHISA SHINOMURA ${ }^{1}$
}

\author{
${ }^{1}$ First Department of Internal Medicine, Sapporo Medical University, Sapporo 060-8543; \\ ${ }^{2}$ The Institute of Medical Science, The University of Tokyo, Tokyo 108-8639, Japan
}

Received July 20, 2010; Accepted September 29, 2010

DOI: $10.3892 / \mathrm{ol} .2010 .197$

\begin{abstract}
Interferon-induced transmembrane protein (IFITM) is reported to be frequently overexpressed in colorectal tumors. This study aimed to determine the usefulness of detecting fecal IFITM messenger RNA (mRNA) by real-time reverse-transcription polymerase chain reaction (RT-PCR) for colorectal cancer (CRC) screening. This pilot study included 21 patients with CRC and 23 healthy controls. Total RNA was isolated from the feces of the patients, and the expression levels of the mRNA of IFITM1, IFITM2 and IFITM3 were measured by real-time RT-PCR to detect CRC. Receiver operating characteristic curves of respective genes were generated, and the area under the curve (AUC), sensitivity and specificity were determined. When the 44 patients were analyzed, the AUCs of fecal IFITM1, IFITM2 and IFITM3 expression analysis were $0.82,0.80$ and 0.65 , respectively. The sensitivities were $67 \%$ [14/21; 95\% confidence interval (CI) 43-85\%], 67\% (14/21; 95\% CI $43-85 \%)$ and $71 \%$ (15/21; 95\% CI 48-89\%), respectively; and the specificities were $96 \%(1 / 23 ; 95 \%$ CI $78-100 \%)$, $96 \%(1 / 23 ; 95 \%$ CI $78-100 \%)$ and $61 \%(9 / 23 ; 95 \%$ CI $39-80 \%)$, respectively. When IFITM1 and IFITM2 were combined, the sensitivity was $86 \%(18 / 21 ; 95 \%$ CI $64-97 \%)$ and the specificity was $96 \%$ (1/23; 95\% CI 78-100\%). The fecal expression analysis of IFITM1 and IFITM2 mRNA by real-time RT-PCR for CRC screening exhibited high specificities, and the sensitivity was further improved by combining IFITM1 and IFITM2.
\end{abstract}

Correspondence to: Dr Nobuki Miyamoto, First Department of Internal Medicine, Sapporo Medical University, South-1, West-16 Chuo-ku, Sapporo 060-8543, Japan

E-mail: nbkmiyamoto1999@yahoo.co.jp

Abbreviations: ACTB, $\beta$-actin; AUC, area under the curve; $\mathrm{CI}$, confidence interval; $\mathrm{CRC}$, colorectal cancer; $\mathrm{Ct}$, cycle threshold; FOBT, fecal occult blood testing; GAPDH, glyceraldehyde-3phosphate dehydrogenase; IFITM, interferon-induced transmembrane protein; mRNA, messenger RNA; PCR, polymerase chain reaction; ROC, receiver operating characteristic; RT, reverse transcription

Key words: colorectal cancer, feces, interferon-induced transmembrane protein, screening, RNA

\section{Introduction}

Colorectal cancer (CRC) is one of the most common types of cancer. Approximately 1 million new cases are diagnosed and approximately 529,000 patients succumb to this type of cancer worldwide each year (1). When this cancer is diagnosed with localized disease, the five-year survival rate following curative surgery is approximately 90\% (2). However, the prognosis worsens with advancing stage, and only $5 \%$ of patients diagnosed with distant metastasis survive for five years. Detection of CRC in the early stage is therefore a key factor for reducing $\mathrm{CRC}$ mortality rates.

Among the various screening tests for CRC, fecal occult blood testing (FOBT) is considered to be the most effective non-invasive screening test. FOBT is convenient and relatively cost-effective (3-6). Guaiac-based FOBT reduces incidence and mortality $(3,7,8)$, but does not exhibit high sensitivity (9-11). Immunochemical FOBT was reported to have a sensitivity of $65.8 \%$ and a specificity of $95 \%$ for detecting CRC (12). Immunochemical FOBT exhibits a higher sensitivity than that of guaiac-based FOBT. However, improvement in the sensitivity of the fecal test for CRC screening is required to reduce mortality rates from this type of cancer.

Numerous screening methods for CRC using fecal DNA are available. Methods using fecal DNA allow for the detection of mutated (13-16), methylated (17-22) or long DNA $(21,23,24)$. Results from various studies on fecal RNA-based analysis by reverse-transcription polymerase chain reaction (RT-PCR) for CRC screening have been reported (25-31). Altered messenger RNA (mRNA) expression of numerous genes has been noted in CRC, but only a few genes have been studied to investigate the usefulness of fecal RNA analysis in CRC detection.

Interferon-induced transmembrane protein (IFITM) mRNA has been found to be overexpressed in CRC tissues compared with expression levels in normal tissues by cDNA microarrays (32). Three homologues (IFITM1, IFITM2 and IFITM3) of the human IFITM gene exist. Frequent upregulation of the IFITM gene expression has been reported to be highly specific to human colorectal carcinogenesis (33).

Quantification by real-time PCR is considered to be useful for determining the optimal cut-off point for discriminating between patients with and without CRC. However, the usefulness of detecting fecal mRNA by real-time RT-PCR for CRC 
Table I. The characteristics of the CRC and control patients.

\begin{tabular}{lcc}
\hline & CRC patients & Control patients \\
\hline No. & 21 & 23 \\
Gender (male/female) & $14 / 7$ & $14 / 9$ \\
Median age (range) & $74(56-94)$ & $61(40-80)$ \\
\hline
\end{tabular}

screening has yet to be studied sufficiently (29). The usefulness of detecting the fecal mRNA of IFITM1, IFITM2 and IFITM3 by real-time RT-PCR for CRC screening was therefore examined.

\section{Materials and methods}

Study design. This study consisted of $21 \mathrm{CRC}$ and 23 control patients (Table I), all of whom underwent colonoscopy. The reasons for performing colonoscopy in the CRC and control patients included positive results of a FOBT test, abdominal pain, anemia, constipation and CRC screening. Stool samples of CRC patients diagnosed with both colonoscopy and histologically were collected prior to surgical resection. The median age of the patients with CRC was 74 years (range 56-94). There were 14 male and 7 female CRC patients. The primary tumor sites were: rectum, 5 patients; sigmoid colon, 7 patients; descending colon, 0 patients; transverse colon, 2 patients; ascending colon, 4 patients; and cecum, 3 patients. The median size of the primary tumors of 14 patients with CRC was $34 \mathrm{~mm}$ (range 10-70) and the size of the tumors of the remaining 7 patients with $\mathrm{CRC}$ was unknown. The tumors were classified according to Dukes' staging, yielding stage A $(n=9)$, and stages B $(n=3), C(n=7)$ and D $(n=2)$ (Table II). A total of 23 control patients (14 male and 9 female) who did not exhibit neoplastic lesions colonoscopically were also included in this study. The median age of the control patients was 61 years (range 40-80). This study was approved by the ethics committees at the institutions in which fecal samples were collected. Oral and written informed consent was obtained from the patients.

Fecal sample collection and RNA isolation. Fecal samples were initially preserved at $-80^{\circ} \mathrm{C}$ within $24 \mathrm{~h}$ following evacuation. Total RNA was extracted from $500 \mathrm{mg}$ of feces without isolating colonocytes using a combination of Isogene (Nippon Gene, Toyama, Japan) and RNeasy kit (Qiagen, Tokyo, Japan) as previously described (26).

cDNA synthesis. The concentration of isolated RNA was measured by NanoDrop 1000 (Thermo Fisher Scientific, Yokohama, Japan). cDNA was synthesized using SuperScript III RNase $\mathrm{H}^{-}$reverse transcriptase (Invitrogen, Tokyo, Japan) with $1000 \mathrm{ng}$ fecal total RNA and $150 \mathrm{ng}$ random primers in a total reaction volume of $18 \mu \mathrm{l}$ according to the manufacturer's instructions.

Table II. The characteristics of the CRC patients.

\begin{tabular}{|c|c|c|c|c|c|}
\hline Patient no. & Age (years) & Gender (male/female) & Location & Size $(\mathrm{cm})$ & Dukes' stage \\
\hline 1 & 63 & M & $\mathrm{R}$ & un & $\mathrm{C}$ \\
\hline 2 & 94 & M & $\mathrm{R}$ & un & B \\
\hline 3 & 57 & M & $\mathrm{S}$ & 3.3 & B \\
\hline 4 & 74 & $\mathrm{~F}$ & $\mathrm{Ce}$ & un & $\mathrm{D}$ \\
\hline 5 & 75 & M & $\mathrm{R}$ & 5.5 & A \\
\hline 6 & 56 & M & $\mathrm{S}$ & un & A \\
\hline 7 & 71 & M & $\mathrm{R}$ & 4.0 & $\mathrm{C}$ \\
\hline 8 & 60 & M & $\mathrm{R}$ & 7.0 & A \\
\hline 9 & 70 & $\mathrm{~F}$ & $\mathrm{~S}$ & 1.3 & A \\
\hline 10 & 85 & M & $\mathrm{T}$ & un & $\mathrm{C}$ \\
\hline 11 & 75 & M & $\mathrm{Ce}$ & un & $\mathrm{C}$ \\
\hline 12 & 85 & M & A & un & $\mathrm{C}$ \\
\hline 13 & 86 & M & $\mathrm{Ce}$ & 1.0 & A \\
\hline 14 & 82 & $\mathrm{~F}$ & $\mathrm{~S}$ & 1.5 & A \\
\hline 15 & 81 & $\mathrm{M}$ & A & 4.0 & A \\
\hline 16 & 70 & $\mathrm{M}$ & $\mathrm{S}$ & 1.5 & A \\
\hline 17 & 70 & $\mathrm{M}$ & $\mathrm{S}$ & 2.0 & A \\
\hline 18 & 65 & $\mathrm{~F}$ & A & 4.0 & $\mathrm{C}$ \\
\hline 19 & 72 & $\mathrm{~F}$ & $\mathrm{~T}$ & 2.5 & $\mathrm{C}$ \\
\hline 20 & 88 & $\mathrm{~F}$ & $\mathrm{~S}$ & 3.5 & B \\
\hline 21 & 80 & $\mathrm{~F}$ & A & 4.0 & $\mathrm{D}$ \\
\hline
\end{tabular}

Ce, cecum; A, ascending; T, transverse; D, descending; S, sigmoid; R, rectum; un, unknown. 
Table III. The primer and probe oligo sequences.

Gene

$\begin{array}{ll}\text { GAPDH } & \text { Forward: GAACGGGAAGCTTGTCATCA } \\ \text { GAPDH } & \text { Reverse: ATCGCCCCACTTGATTTTG } \\ \text { GAPDH } & \text { Probe: FAM-CCCATCACCATCTTCCAGGAGCGAGA-TAMRA } \\ \text { ACTB } & \text { Forward: CCTCGCCTTTGCCGATCC } \\ \text { ACTB } & \text { Reverse: CATGCCGGAGCCGTTGTC } \\ \text { ACTB } & \text { Probe: FAM-CGTCCACACCCGCCGCCAGC-TAMRA } \\ \text { IFITM1 } & \text { Forward: TCGCCTACTCCGTGAAGTCT } \\ \text { IFITM1 } & \text { Reverse: TGTCACAGAGCCGAATACCA } \\ \text { IFITM1 } & \text { Probe: FAM-ATGCCTCCACCGCCAAGTGCCT-TAMRA } \\ \text { IFITM2 } & \text { Forward: TGTATCCCACGTACTCTATCTTCC } \\ \text { IFITM2 } & \text { Reverse: GGACAGGGCGAGGAATGG } \\ \text { IFITM2 } & \text { Probe: FAM-TGGAGTAAGTGGAATACAGGTCAAGGGCAG-TAMRA } \\ \text { IFITM3 } & \text { Forward: CTGAGAACCATCCCAGTAACCC } \\ \text { IFITM3 } & \text { Reverse: ACTGTTGACAGGAGAGAAGAAGG } \\ \text { IFITM3 } & \text { Probe: FAM-CATGGTGTCCAGCGAAGACCAGCGG-TAMRA }\end{array}$

GAPDH, glyceraldehyde-3-phosphate dehydrogenase; ACTB, $\beta$-actin; IFITM, interferon-induced transmembrane protein; FAM, carboxyfluorescein-aminohexyl amidite; TAMRA, tetramethylrhodamine.

Real-time polymerase chain reaction. Amplification and detection were performed by real-time PCR with a Taq Man probe. The expression levels of glyceraldehyde-3-phosphate dehydrogenase (GAPDH), $\beta$-actin (ACTB), IFITM1, IFITM2 and IFITM3 were measured. The sequences of the PCR primers and probes are listed in Table III. cDNA $(2 \mu \mathrm{l})$ was used as templates in each reaction. The reaction mixture consisted of templates, $10 \mu \mathrm{l}$ of QuantiTect Multiplex PCR kit (Qiagen, Tokyo, Japan), $0.40 \mu \mathrm{M}$ of forward and reverse primers and $0.20 \mu \mathrm{M}$ of the probe in a total reaction volume of $20 \mu \mathrm{l}$. The real-time PCR reaction was performed with precycling heat activation at $95^{\circ} \mathrm{C}$ for $10 \mathrm{~min}$, followed by 50 cycles of denaturation at $95^{\circ} \mathrm{C}$ for $15 \mathrm{sec}$ and annealing/ extension at $60^{\circ} \mathrm{C}$ for $60 \mathrm{sec}$ in an Applied Biosystems 7500 sequence detection system (Life Technologies, Tokyo, Japan).

Statistical analysis. In the fecal RNA analysis used for detecting CRC, when an amplification curve crossed the threshold line within the end of definite cycles, the expression of the gene was interpreted as positive, and multiple pairs of sensitivities and specificities were determined for each gene. The receiver operating characteristic (ROC) curve was created from pairs of sensitivities and specificities, and the area under the curve (AUC) was calculated (34). The optimal sensitivity and specificity were determined using the Youden index: $($ Youden index $)=$ maximum $($ sensitivity + specificity -1$)$. The sensitivities and specificities were estimated relative to the results of the colonoscopy in the usual manner; $95 \%$ confidence interval (CI) for each of the estimated parameters was based on the exact binominal distribution. $\mathrm{P}<0.05$ was considered to be statistically significant. The reported P-values were evaluated by a two-sided test.

\section{Results}

RNA concentration. The mean concentrations of RNA extracted from the feces of the $21 \mathrm{CRC}$ and 23 control patients were $876 \mathrm{ng} / \mu \mathrm{l}$ (range 105-3,333) and 1,044 $\mathrm{ng} / \mu \mathrm{l}$ (range 105-2,000), respectively. No significant difference was found between RNA concentrations in the group of CRC patients and the group of control patients $(\mathrm{P}=0.40)$.

Detection of GAPDH and ACTB $m R N A$. The detection rates of GAPDH were $91 \%(19 / 21)$ in the CRC patients and $100 \%$ $(23 / 23)$ in the control patients. No significant difference was noted between the detection rates of GAPDH in the CRC and control patients $(\mathrm{P}=0.2)$. The cycle threshold $(\mathrm{Ct})$ values of GAPDH in the CRC patients were significantly smaller than those in the control patients $(\mathrm{P}=0.02)$. The detection rates of ACTB were $76 \%(16 / 21)$ in the CRC patients and $91 \%$ $(21 / 23)$ in the control patients. No significant difference was found between the detection rates of ACTB in the CRC and control patients $(\mathrm{P}=0.23)$. The $\mathrm{Ct}$ values of $\mathrm{ACTB}$ in $\mathrm{CRC}$ patients were significantly smaller than those in the control patients $(\mathrm{P}=0.003)$.

Sensitivities and specificities of the gene expression analysis. When the 44 cases were analyzed, the AUCs of fecal IFITM1, IFITM2 and IFITM3 expression analysis for CRC were 0.82, 0.80 and 0.65 , respectively (Fig. 1). The sensitivities were $67 \%$ (14/21; 95\% CI 43-85\%), 67\% (14/21; 95\% CI 43-85\%) and $71 \%(15 / 21 ; 95 \%$ CI $48-89 \%)$, respectively. The specificities were $96 \%(1 / 23$; $95 \%$ CI $78-100 \%), 96 \%(1 / 23 ; 95 \%$ CI $78-100 \%)$ and $61 \%(9 / 23 ; 95 \%$ CI $39-80 \%)$, respectively. The Youden index values were $0.62,0.62$ and 0.32 , respectively (Table IV). 
Table IV. The sensitivities and specificities of fecal RNA expression analysis.

\begin{tabular}{|c|c|c|c|c|c|c|c|}
\hline Expression & Gene & No. & $\begin{array}{l}\text { sitivity } \\
\%(95 \% \mathrm{CI})\end{array}$ & No. & $\begin{array}{l}\text { cificity } \\
\%(95 \% \mathrm{CI})\end{array}$ & $\begin{array}{l}\text { Threshold } \\
\text { (cycles) }\end{array}$ & $\begin{array}{l}\text { Youden } \\
\text { index }\end{array}$ \\
\hline \multicolumn{8}{|l|}{ ALL } \\
\hline & IFITM1 & $14 / 21$ & $67(43-85)$ & $1 / 23$ & $96(78-100)$ & 45 & 0.62 \\
\hline & IFITM2 & $14 / 21$ & $67(43-85)$ & $1 / 23$ & $96(78-100)$ & 39 & 0.62 \\
\hline & IFITM3 & $15 / 21$ & $71(48-89)$ & $9 / 23$ & $61(39-80)$ & 38 & 0.32 \\
\hline & IFITM1+2 & $18 / 21$ & $86(64-97)$ & $1 / 23$ & $96(78-100)$ & $\begin{array}{l}\text { IFITM1:45 } \\
\text { IFITM2:35 }\end{array}$ & 0.81 \\
\hline \multicolumn{8}{|l|}{ GAPDH (+) } \\
\hline & IFITM1 & $14 / 19$ & $74(49-91)$ & $1 / 23$ & $96(78-100)$ & 45 & 0.69 \\
\hline & IFITM2 & $14 / 19$ & $74(49-91)$ & $1 / 23$ & $96(78-100)$ & 39 & 0.69 \\
\hline & IFITM3 & $15 / 19$ & 79 (54-94) & $9 / 23$ & $61(39-80)$ & 38 & 0.40 \\
\hline & IFITM1+2 & $18 / 19$ & $95(74-100)$ & $1 / 23$ & $96(78-100)$ & $\begin{array}{l}\text { IFITM1:45 } \\
\text { IFITM2:35 }\end{array}$ & 0.90 \\
\hline \multicolumn{8}{|l|}{$\operatorname{ACTB}(+)$} \\
\hline & IFITM1 & $12 / 16$ & $75(48-93)$ & $1 / 21$ & $95(76-100)$ & 44 & 0.70 \\
\hline & IFITM2 & $14 / 16$ & $88(62-99)$ & $1 / 21$ & $95(76-100)$ & 39 & 0.83 \\
\hline & IFITM3 & $14 / 16$ & $88(62-99)$ & $7 / 21$ & $67(43-85)$ & 38 & 0.54 \\
\hline & IFITM1+2 & $16 / 16$ & $100(79-100)$ & $0 / 21$ & $100(84-100)$ & $\begin{array}{l}\text { IFITM1:44 } \\
\text { IFITM2:35 }\end{array}$ & 1.00 \\
\hline
\end{tabular}

No., the number of positive/total; +, positive gene expression. ALL, all 44 cases.
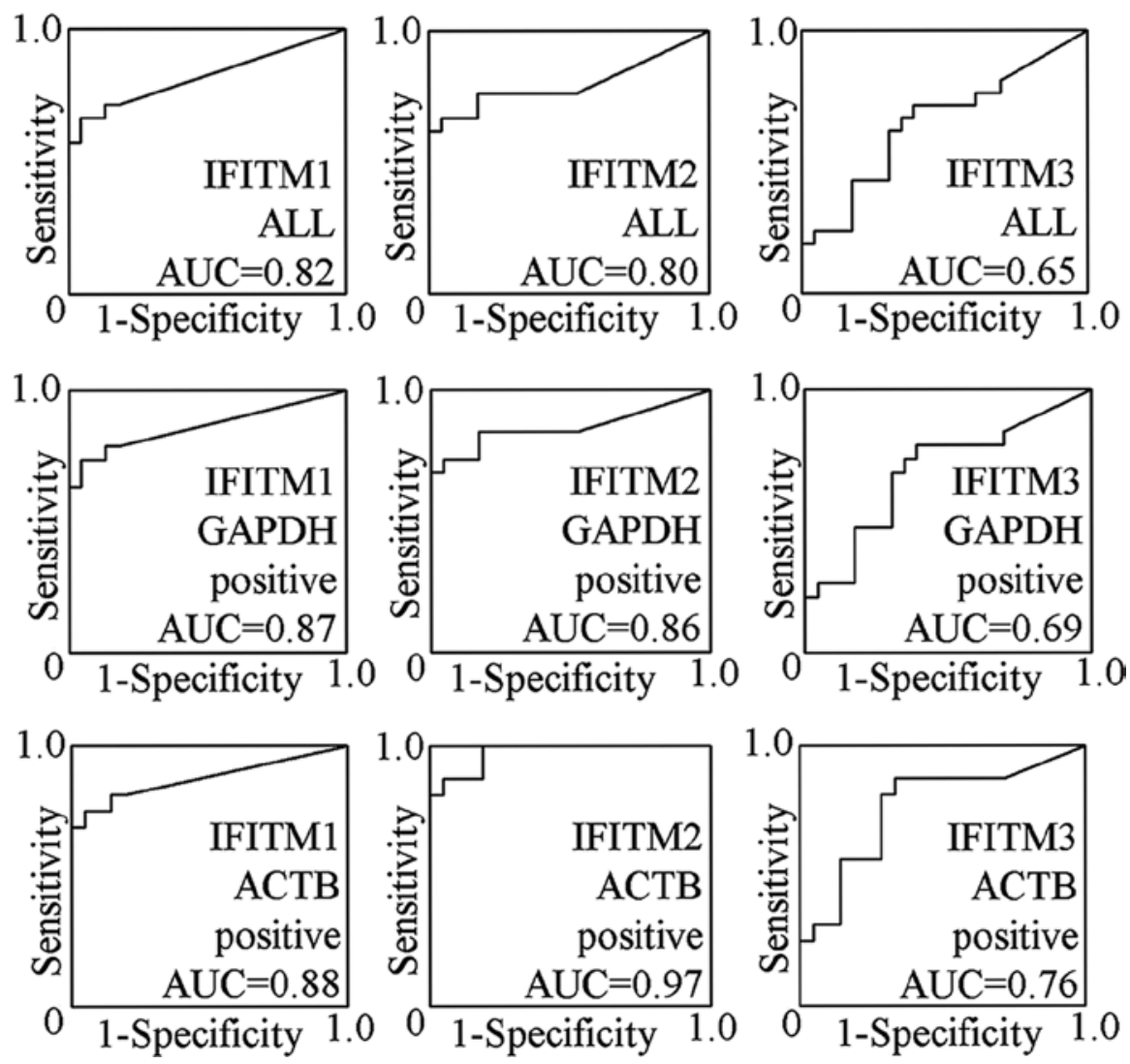

Figure 1. ROC curves and AUC of fecal RNA expression analysis. The markers were determined in all 44 cases (21 CRC and 23 control patients), in the cases whose fecal mRNA expression of GAPDH was positive (19 CRC and 23 control patients) and in the cases whose fecal mRNA expression of ACTB was positive (16 CRC and 21 control patients). ALL, all 44 cases. 
When 42 cases were analyzed in which GAPDH mRNA was detected, the AUCs of fecal IFITM1, IFITM2 and IFITM3 expression analysis for CRC were $0.87,0.86$ and 0.69 , respectively (Fig. 1). The sensitivities were 74\% (14/19; 95\% CI 49-91\%), 74\% (14/19; 95\% CI 49-91\%) and 79\% (15/19; 95\% CI $54-94 \%)$, respectively. The specificities were $96 \%(1 / 23 ; 95 \%$ CI $78-100 \%), 96 \%(1 / 23$; $95 \%$ CI $78-100 \%)$ and $61 \%(9 / 23$; 95\% CI 39-80\%), respectively, and the Youden index values were $0.69,0.69$ and 0.40 , respectively (Table IV).

When 37 cases were analyzed in which ACTB mRNA was detected, the AUCs of fecal IFITM1, IFITM2 and IFITM3 expression analysis for CRC were $0.88,0.97$ and 0.76 , respectively (Fig. 1). The sensitivities were $75 \%(12 / 16 ; 95 \% \mathrm{CI}$ $48-93 \%), 88 \%$ (14/16; 95\% CI 62-99\%) and 88\% (14/16; 95\% CI 62-99\%), respectively. The specificities were $95 \%(1 / 21$; 95\% CI 76-100\%), 95\% (1/21; 95\% CI 76-100\%) and 67\% (7/21; 95\% CI 43-85\%), respectively, and the Youden index values were $0.70,0.83$ and 0.54 , respectively (Table IV).

The sensitivities of fecal IFITM1, IFITM2 and IFITM3 expression analysis of the patients with Dukes' A+B were 58\% (7/12; 95\% CI 30-87\%), 67\% (8/12; 95\% CI 38-95\%) and 67\% (8/12; 95\% CI 38-95\%), respectively, and the patients with Dukes' C+D exhibited 78\% (7/9; 95\% CI 45-100\%), 67\% (6/9; 95\% CI 34-99\%) and 78\% (7/9; 95\% CI 45-100\%), respectively. Therefore, no significant difference was found in the sensitivities between the two groups. There was also no significant difference in the sensitivities of fecal IFITM1, IFITM2 and IFITM3 expression analysis regarding gender, tumor location, tumor size, RNA concentration or GAPDH expression. The sensitivities of fecal IFITM2 and IFITM3 expression analysis of the group in which ACTB expression was positive were significantly higher than those of the group in which ACTB expression was negative $(\mathrm{P}=0.001$ and $\mathrm{P}=0.01$, respectively), although no significant difference was found in the sensitivity of fecal IFITM1 expression analysis between the two groups.

Combination of IFITM1 and IFITM2. The AUCs of IFITM1 and IFITM2 were larger than that of IFITM3. Therefore, we calculated the sensitivities and specificities for the combination of IFITM1 and IFITM2 (fecal IFITM1+2 expression analysis). When analyzed for all 44 cases, the sensitivity and specificity of fecal IFITM1+2 expression analysis were found to be $86 \%(18 / 21 ; 95 \%$ CI $64-97 \%)$ and $96 \%(1 / 23 ; 95 \%$ CI $78-100 \%)$. When analyzed for cases in which GAPDH mRNA was detected, the sensitivity and specificity were 95\% (18/19; 95\% CI $74-100 \%)$ and $96 \%$ (1/23; 95\% CI $78-100 \%)$. When analyzed for cases in which ACTB mRNA was detected, the sensitivity and specificity were $100 \%(16 / 16 ; 95 \%$ CI $79-100 \%)$ and $100 \%$ (0/21; 95\% CI 84-100\%) (Table IV).

The sensitivity of fecal IFITM1+2 analysis of patients with Dukes' A+B and that of patients with Dukes' C+D were 83\% (10/12; 95\% CI 55-100\%) and 89\% (8/9; 95\% CI 56-100\%), respectively, and no significant difference was noted in the sensitivities between the two groups. There was also no significant difference in the sensitivities of fecal IFITM1+2 expression analysis with regards to gender, tumor location, tumor size or RNA concentration. The sensitivity of fecal IFITM1+2 expression analysis of the group in which GAPDH expression was positive was significantly higher than that of the group in which GAPDH expression was negative $(\mathrm{P}=0.01)$, and the sensitivity of the group in which ACTB expression was positive was significantly higher than that of the group in which ACTB expression was negative $(\mathrm{P}=0.01)$.

\section{Discussion}

Results of numerous studies on fecal DNA-based analysis for CRC screening have been reported. A fecal DNA panel consisting of 21 mutations exhibited $51.6 \%$ sensitivity and 94.4\% specificity (11). Since CRC cells undergo diverse genetic changes, it is difficult to detect CRC with a high sensitivity by using only fecal DNA-based mutational analysis.

Fecal methylation analysis of the vimentin gene provided sensitivity and specificity of 72.5 and $86.9 \%$, respectively, and the combination of vimentin methylation plus a DNA integrity assay resulted in $87.5 \%$ sensitivity and $82 \%$ specificity (21). In another study, a fecal SFRP2 methylation assay for CRC screening exhibited $77-90 \%$ sensitivity and $77 \%$ specificity (20). In the fecal methylation analysis, it is crucial to decrease the effect of methylation with aging to improve the specificity.

The number of studies on fecal RNA-based analysis for CRC screening that are currently available are fewer than those on fecal DNA-based analysis. The fecal RNA expression analysis of PTGS2 by semi-quantitative RT-PCR was reported to have sensitivities of 50-90\% and specificities of $93-100 \%$ in previous studies $(22,26,30)$. Semi-quantitative RT-PCR was used in the majority of previous studies on fecal RNA expression analysis for CRC screening, while real-time RT-PCR was used in only a few studies (29). Real-time PCR has a number of advantages over semi-quantitative PCR, including high speed, reduction of contamination and a high level of reproducibility, from the viewpoint of a laboratory test. In the present study, the sensitivity and specificity of fecal IFITM1+2 expression analysis determined in all 44 cases were 86 and 96\%, respectively. We showed that CRC is potentially detected with a high sensitivity and specificity by fecal RNA expression analysis using real-time RT-PCR.

The quantitation of templates by real-time RT-PCR allowed us to generate ROC curves for the fecal mRNA expression analysis, compare the AUCs, and determine the cut-off points at which optimal sensitivities and specificities are achieved. Real-time RT-PCR is considered to be effective in determining the optimal cut-off points efficiently when studying the usefulness of fecal mRNA expression analysis by RT-PCR for CRC screening.

Up-regulation of the IFITM gene was considered to be an early event in $\beta$-catenin intestinal tumorigenesis (33). No significant difference was noted in the sensitivities of fecal IFITM1, IFITM2, IFITM3 and IFITM1+2 expression analysis between the group of CRC patients without metastasis (Dukes' $\mathrm{A}+\mathrm{B}$ ) and the group of CRC patients with metastasis (Dukes' C+D). Therefore, the fecal IFITM expression analysis appears to be useful in the early detection of CRC.

No significant difference was found in sensitivities regarding tumor size and location. However, the sensitivity for tumors of less than $34 \mathrm{~mm}$ in diameter were lower than that for tumors of more than $34 \mathrm{~mm}$ in diameter. The sensitivity for tumors located on the right side of the colon were lower than that for tumors located on the left side of the colon. A larger study is therefore required to clarify the differences in 
sensitivities of fecal mRNA expression analysis for CRC with regards to tumor size and/or location.

When analyzed for cases in which the mRNA of the housekeeping gene was detected, AUCs were found to be larger than AUCs when analyzed for all 44 cases. Moreover, the sensitivity of fecal IFITM1+2 expression analysis in the group in which the mRNA of the housekeeping gene was not detected was significantly lower than that in the group in which the mRNA of the housekeeping gene was detected. Fecal IFITM mRNA-negative CRC cases appear to include not only cases in which IFITM mRNA was not expressed in their tumors but also ones in which human RNA as templates of RT-PCR was insufficient in their fecal samples.

In conclusion, detection of fecal mRNA of IFITM1 and IFITM2 had larger AUCs than that of IFITM3, and the sensitivity was improved by combining IFITM1 and IFITM2. Since the number of cases analyzed in the present study was limited, a larger study is imperative in order to assess sensitivity and specificity. However, the results of the present study suggest the usefulness of detecting the fecal mRNA of IFITM1 and IFITM2 by real-time RT-PCR for CRC screening.

\section{Acknowledgements}

Support by Grants-in-Aid for Scientific Research from the Ministry of Education, Culture, Sports, Science and Technology of Japan (to H.Y., K.I. and Y.S.)

\section{References}

1. Parkin DM, Bray F, Ferlay J and Pisani P: Global cancer statistics, 2002. CA Cancer J Clin 55: 74-108, 2005.

2. Pfister DG, Benson AB III and Somerfield MR: Clinical practice. Surveillance strategies after curative treatment of colorectal cancer. N Engl J Med 350: 2375-2382, 2004.

3. Mandel JS, Church TR, Bond JH, et al: The effect of fecal occult-blood screening on the incidence of colorectal cancer. N Engl J Med 343: 1603-1607, 2000.

4. Winawer S, Fletcher R, Rex D, et al: Colorectal cancer screening and surveillance: clinical guidelines and rationale-update based on new evidence. Gastroenterology 124: 544-560, 2003

5. Smith A, Young GP, Cole SR and Bampton P: Comparison of a brush-sampling fecal immunochemical test for hemoglobin with a sensitive guaiac-based fecal occult blood test in detection of colorectal neoplasia. Cancer 107: 2152-2159, 2006.

6. Levi Z, Rozen P, Hazazi R, et al: A quantitative immunochemical fecal occult blood test for colorectal neoplasia. Ann Intern Med 146: 244-255, 2007.

7. Kronborg O, Fenger C, Olsen J, Jørgensen OD and Søndergaard O: Randomised study of screening for colorectal cancer with faecaloccult-blood test. Lancet 348: 1467-1471, 1996.

8. Hardcastle JD, Chamberlain JO, Robinson $\mathrm{MH}$, et al: Randomised controlled trial of faecal-occult-blood screening for colorectal cancer. Lancet 348: 1472-1477, 1996.

9. Lieberman DA and Weiss DG: One-time screening for colorectal cancer with combined fecal occult-blood testing and examination of the distal colon. N Engl J Med 345: 555-560, 2001.

10. Sung JJ, Chan FK, Leung WK, et al: Screening for colorectal cancer in Chinese: comparison of fecal occult blood test, flexible sigmoidoscopy, and colonoscopy. Gastroenterology 124 608-614, 2003

11. Imperiale TF, Ransohoff DF, Itzkowitz SH, Turnbull BA and Ross ME: Fecal DNA versus fecal occult blood for colorectalcancer screening in an average-risk population. N Engl J Med 351: 2704-2714, 2004.

12. Morikawa T, Kato J, Yamaji Y, Wada R, Mitsushima T and Shiratori Y: A comparison of the immunochemical fecal occult blood test and total colonoscopy in the asymptomatic population. Gastroenterology 129: 422-428, 2005.
13. Ahlquist DA, Skoletsky JE, Boynton KA, et al: Colorectal cancer screening by detection of altered human DNA in stool: feasibility of a multitarget assay panel. Gastroenterology 119: 1219-1227, 2000.

14. Dong SM, Traverso G, Johnson C, et al: Detecting colorectal cancer in stool with the use of multiple genetic targets. J Natl Cancer Inst 93: 858-865, 2001.

15. Traverso G, Shuber A, Levin B, et al: Detection of APC mutations in fecal DNA from patients with colorectal tumors. N Engl J Med 346: 311-320, 2002.

16. Diehl F, Schmidt K, Durkee KH, et al: Analysis of mutations in DNA isolated from plasma and stool of colorectal cancer patients. Gastroenterology 135: 489-498, 2008

17. Lenhard K, Bommer GT, Asutay S, et al: Analysis of promoter methylation in stool: a novel method for the detection of colorectal cancer. Clin Gastroenterol Hepatol 3: 142-149, 2005.

18. Chen WD, Han ZJ, Skoletsky J, et al: Detection in fecal DNA of colon cancer-specific methylation of the nonexpressed vimentin gene. J Natl Cancer Inst 97: 1124-1132, 2005.

19. Zou H, Harrington J, Rego RL and Ahlquist DA: A novel method to capture methylated human DNA from stool: implications for colorectal cancer screening. Clin Chem 53: 1646-1651, 2007.

20. Müller HM, Oberwalder M, Fiegl H, et al: Methylation changes in faecal DNA: a marker for colorectal cancer screening? Lancet 363: 1283-1285, 2004.

21. Itzkowitz SH, Jandorf L, Brand R, et al: Improved fecal DNA test for colorectal cancer screening. Clin Gastroenterol Hepatol 5: 111-117, 2007.

22. Leung WK, To KF, Man EP, et al: Detection of hypermethylated DNA or cyclooxygenase-2 messenger RNA in fecal samples of patients with colorectal cancer or polyps. Am J Gastroenterol 102: 1070-1076, 2007.

23. Boynton KA, Summerhayes IC, Ahlquist DA and Shuber AP: DNA integrity as a potential marker for stool-based detection of colorectal cancer. Clin Chem 49: 1058-1065, 2003.

24. Zou H, Harrington JJ, Klatt KK and Ahlquist DA: A sensitive method to quantify human long DNA in stool: relevance to colorectal cancer screening. Cancer Epidemiol Biomarkers Prev 15: 1115-1119, 2006.

25. Yamao T, Matsumura Y, Shimada Y, et al: Abnormal expression of CD44 variants in the exfoliated cells in the feces of patients with colorectal cancer. Gastroenterology 114: 1196-1205, 1998.

26. Kanaoka S, Yoshida K, Miura N, Sugimura $H$ and Kajimura M: Potential usefulness of detecting cyclooxygenase 2 messenger RNA in feces for colorectal cancer screening. Gastroenterology 127: 422-427, 2004.

27. Lagerholm S, Lagerholm S, Dutta S and Nair P: Non-invasive detection of c-myc p64, c-myc p67 and c-erbb-2 in colorectal cancer. Scand J Gastroenterol 40: 1343-1350, 2005.

28. Yajima S, Ishii M, Matsushita $\mathrm{H}$, et al: Expression profiling of fecal colonocytes for RNA-based screening of colorectal cancer. Int J Oncol 31: 1029-1037, 2007.

29. Koga Y, Yasunaga M, Moriya Y, et al: Detection of colorectal cancer cells from feces using quantitative real-time RT-PCR for colorectal cancer diagnosis. Cancer Sci 99: 1977-1983, 2008.

30. Takai T, Kanaoka S, Yoshida K, et al: Fecal cyclooxygenase 2 plus matrix metalloproteinase $7 \mathrm{mRNA}$ assay as a marker for colorectal cancer screening. Cancer Epidemiol Biomarkers Prev 18: 1888-1893, 2009.

31. Yu YJ, Majumdar AP, Nechvatal JM, et al: Exfoliated cells in stool: a source for reverse transcription-PCR-based analysis of biomarkers of gastrointestinal cancer. Cancer Epidemiol Biomarkers Prev 17: 455-458, 2008.

32. Kitahara O, Furukawa Y, Tanaka T, et al: Alterations of gene expression during colorectal carcinogenesis revealed by cDNA microarrays after laser-capture microdissection of tumor tissues and normal epithelia. Cancer Res 61: 3544-3549, 2001.

33. Andreu P, Colnot S, Godard C, et al: Identification of the IFITM family as a new molecular marker in human colorectal tumors. Cancer Res 66: 1949-1955, 2006.

34. Akobeng AK: Understanding diagnostic tests 3: receiver operating characteristic curves. Acta Pediatrica 96: 644-647, 2007. 